\title{
RELAÇÃO ENTRE TESTES FUNCIONAIS E PLATAFORMA DE FORÇA NAS MEDIDAS DE EQUILÍBRIO EM ATLETAS
}

\author{
RELATIONSHIP BETWEEN FUNCTIONAL TESTS AND FORCE PLATFORM MEASUREMENTS \\ IN ATHLETES'BALANCE \\ RELACIÓN ENTRE PRUEBAS FUNCIONALES Y PLATAFORMA DE FUERZAS EN LAS MEDIDAS DE \\ BALANCE DE LOS ATLETAS
}

ARTIGO ORIGINAL

\author{
Lucas Maciel Rabello ${ }^{1,4}$ \\ (Fisioterapeuta) \\ Christiane de Souza Guerino \\ Macedo ${ }^{3}$ (Fisioterapeuta) \\ Márcio Rogério de \\ Oliveira' (Fisioterapeuta) \\ João Henrique Fregueto ${ }^{2}$ \\ (Graduando em Fisioterapia) \\ Mariana Zingari Camargo' \\ (Fisioterapeuta) \\ Luana Deguchi Lopes ${ }^{1}$ \\ (Graduando em Fisioterapia) \\ Leonardo Shigaki ${ }^{1}$ \\ (Fisioterapeuta) \\ Cynthia Gobbi ${ }^{1}$ (Fisioterapeuta) \\ André Wilson Gil ${ }^{1}$ (Fisioterapeuta) \\ Camila Kamuza² (Fisioterapeuta) \\ Rubens Alexandre da Silva Jr. ${ }^{1,2}$ \\ (Fisioterapeuta) \\ 1. Laboratório de Avaliação \\ Funcional e Performance \\ Motora Humana (LAFUP), da \\ Universidade Norte do Paraná \\ (UNOPAR), Londrina, PR, Brasil. \\ 2. Universidade Norte do Paraná \\ (UNOPAR), Londrina PR, Brasil. \\ 3. Departamento de Fisioterapia, \\ Universidade Estadual de Londrina \\ (UEL), Londrina, PR, Brasil. \\ 4. Faculdade de Educação e Meio \\ Ambiente, Ariquemes RO, Brasil.
}

\section{Correspondência:}

Rubens Alexandre da Silva Jr., Centro de Pesquisa em Ciências da Saúde. Coordenador LAFUP, UNOPAR (KROTON EDUCATION), Av. Paris, 675 - Jd. Piza, 86041-140. Londrina, PR, Brasil.

rubens@kroton.com.br

\section{RESUMO}

Introdução: Poucos estudos correlacionaram as medidas de equilíbrio entre os principais testes funcionais e a plataforma de força em atletas. Objetivo: Determinar a relação entre três testes funcionais de equilíbrio com as principais medidas da plataforma de força em atletas. Método: Quinze atletas do sexo feminino praticantes de futebol de salão (futsal) e handebol, com idades entre 13 a 17 anos participaram do estudo. As atletas realizaram três testes funcionais: 1) Star excursion balance test, 2) Salto lateral e 3) Figura em oito; além de um teste em apoio unipodal sobre uma plataforma de força com os olhos abertos durante 30 segundos. Os parâmetros do centro de pressão dos pés (COP) foram calculados por análise estabilográfica. Para todos os testes (funcionais e plataforma), três tentativas foram realizadas e a média foi retida para as análises. Resultados: As correlações entre os testes funcionais e a plataforma de força foram de $r=0,01$ a -0,69 (fraca a moderada), dependendo da variável do COP. As melhores correlações $(r=-0,69)$ foram encontradas entre o Star Excursion Balance Test de alcance e a área do COP, indicando que quanto maior a distância alcançada, melhor o equilíbrio. Segundo, uma correlação de fraca a moderada foi encontrada entre o teste da figura em oito e a área do COP (p. ex: $r=0.43$ ). O teste de salto lateral não apresentou boa correlação com os obtidos na plataforma de força. Conclusão: Este estudo demonstrou que para a avaliação do equilíbrio em atletas de futsal e handebol feminino o teste Star Excursion Balance Test pode ser usados na ausência da plataforma de força para identificação de possíveis déficits de equilíbrio.

Palavras-chave: atletas, postura, fisioterapia, reabilitação, esporte.

\section{ABSTRACT}

Introduction: Few studies have correlated the balance measurements based in functional tests and force platform in athletes. Objective: to determine the relationship between three functional balance tests with the main measurements of the force platform in athletes. Methodology: Fifteen female athletes, indoor soccer and handball players, aged between 13 and 17 years, participated of this study. The athletes performed tree functional tests: 1) the star excursion balance test, 2) the lateral jump, and 3) the figure eight test; as well as the one leg-stance test on a force platform with the eyes open for 30 seconds. The parameters of center of pressure (COP) of the foot were calculated by stabilographic analysis. For all the tests (functional and platform), tree trials attempts were made, and the mean value retained for the analyses. Results: The correlations between the functional tests and the force platform were from $r=0.01$ to -0.69 (weak to moderate), depending on the COP variable. The best correlations ( $r=-0.69)$ were found between the star excursion balance test and the COP area, indicating that when a greater distance was reached, the better the postural balance. Secondly, a weak to moderate correlation was found between the figure eight test and COP area (e.g. $r=0.43$ ). The side hop test did not present good correlation with the values obtained on the force platform. Conclusion: This study shows that to evaluate balance in athletes practicing indoor soccer and handball, the Star excursion balance test may be used in the absence of a force platform, to identify possible balance deficits.

Keywords: Keywords: athletes, posture, physical therapy, rehabilitation, sports.

\section{RESUMEN}

Introducción: Pocos estudios han correlacionado las medidas de equilibrio entre las principales pruebas funcionales y la plataforma de fuerza en los atletas. Objetivo: Determinar la relación entre tres pruebas de equilibrio funcionales con las principales medidas de la fuerza en la plataforma de los atletas. Método: Quince atletas del sexo femenino que practican el fútbol de salón y balonmano, entre 13 y 17 años participaron en el estudio. Los atletas realizaron tres pruebas funcionales: 1) Star excursion balance test, 2) salto lateral y 3) salto figura ocho, además de una prueba unipodal sobre una plataforma de fuerza con los ojos abiertos durante 30 segundos. Los parámetros del centro de la presión de los pies (COP) se calcularon por análisis estabilográfica. Para todas las pruebas (funcionales y plataforma), se llevaron a cabo tres ensayos y el promedio fue utilizado para su análisis. Resultados: Las correlaciones entre las pruebas funcionales y la plataforma de fuerza fueron $r=0,01$ a -0,69 (leve a moderada), en 
función de la variable COP. La mejor correlación $(r=-0,69)$ se encontró entre el rango de la prueba Star Excursion Balance y el área del COP, lo que indica que cuanto mayor es la distancia que se logra, mejor es el equilibrio. En segundo lugar, se encontró correlación débil a moderada entre la prueba en figura de ocho y el área del COP (por ejemplo, $r=0,43$ ). La prueba de salto lateral mostró una buena correlación con las pruebas en la plataforma de fuerza. Conclusión: Este estudio demostró que para la evaluación del equilibrio de los atletas del sexo femenino de fútbol de salón y balonmano, el Star excursion balance test se puede utilizar en la ausencia de una plataforma de fuerza para identificar posibles deficiencias en el equilibrio.

Palabras clave: atletas, postura, fisioterapia, rehabilitación, deportes.

\section{INTRODUÇÃO}

Os atletas possuem características morfológicas e fisiológicas específicas e estão expostos a um conjunto de exigências físicas inerentes às tarefas motoras presentes em cada modalidade ${ }^{1}$. Durante a prática esportiva é comum à ocorrência de lesões musculoesqueléticas, principalmente em membros inferiores, as quais podem causar limitações físicas importantes como déficit do equilíbrio postural.

O equilíbrio postural é multifacetado e ordenado pela execução do controle motor na ação integrada de três sistemas: visual, vestibular e somatossensorial associados aos estímulos proprioceptivos ${ }^{2}$ Biomecanicamente, o equilíbrio postural pode ser definido como a manutenção do centro de massa do corpo associado dentro da base de suporte delimitada entre os dois pés ${ }^{3}$. Este processo requer ajustes constantes no sistema neuromuscular para adequado equilíbrio dos segmentos corporais ${ }^{4}$.

Os testes de estabilidade articular tais como Figura em oito e Star Excursion Balance Test (SEBT) são geralmente preconizados na prevenção de lesões e no desempenho físico de atletas 5 . Contudo, outro recurso de alta tecnologia comumente utilizado é a plataforma de força, considerado como padrão-ouro para avaliação do equilíbrio postural, que quantitativamente mensuram as oscilações do corpo durante diferentes ações (estática ou dinâmica) 4,6. Em determinados testes funcionais, de caráter mais simples, utiliza-se apenas o tempo de execução da tarefa como domínio de medida7,8, o que pode limitar as conclusões dos resultados principalmente na avaliação dos mecanismos biomecânicos e neuromusculares de controle postural.

Em relação à plataforma de força, são preconizados como domínios de avaliação a informação temporal e a frequência, todas provenientes do deslocamento da área do centro de pressão (COP) dos pés 9 . O COP e suas medidas derivadas de velocidade e frequência são considerados como principais parâmetros de avaliação do controle postural. O COP pode ser definido como a média ponderada de todas as pressões exercidas na área da superfície de contato com o solo ${ }^{9}$ e seu deslocamento refere-se à habilidade do sistema de controle postural manter o centro de massa do corpo dentro dos limites fisiológicos de sustentação dos pés.

Algumas evidências sugerem que testes funcionais não são considerados medidas diretas para investigar e diagnosticar os reais déficits de controle postural6,10. Entretanto, testes funcionais são amplamente utilizados para avaliação da performance funcional de atletas, antes ou após lesão dos membros inferiores ${ }^{8,11}$. Porém, poucos estudos têm correlacionado estes dois métodos de avaliação (testes funcionais e plataforma de força) para analisar a relação entre eles no que concerne às medidas de equilíbrio postural em atletas; o que tem sido apontado são estudos em idosos os quais são limitados para a generalização para a população estudada ${ }^{12}$

Dessa maneira, o objetivo do presente estudo foi determinar a relação entre três principais testes funcionais de estabilidade articular com as principais medidas de equilíbrio da plataforma de força em atletas.

\section{MÉTODOS}

Tratou-se de um estudo analítico do tipo transversal. A amostra do presente estudo foi recrutada por conveniência e de maneira voluntária, sendo formada por 15 atletas saudáveis do sexo feminino, praticantes da modalidade de futebol de salão (futsal) e handebol. As atletas possuíam idade entre 13 a 17 anos, deveriam estar em treinamento esportivo regular no mínimo três dias por semana, participar de competições estaduais e/ou nacionais e não apresentar nenhum tipo lesão neurológica ou ortopédica incapacitante, bem como cirurgias no aparelho locomotor.

O termo de consentimento livre e esclarecido foi assinado pelos pais ou responsável das atletas. O projeto foi aprovado pelo Comitê de Ética em Pesquisa da Instituição (PP/0231/10).

As avaliações ocorreram no Laboratório de Avaliação Funcional e Performance Motora Humana (LAFUP), da Universidade Norte do Paraná (UNOPAR) Brasil. Inicialmente as atletas preencheram uma ficha de avaliação contendo dados demográficos da amostra (idade, peso, altura, dominância, modalidade esportiva, frequência de atividade física e esportiva, presença ou não de queixa de instabilidade em articulação do tornozelo) e em seguida realizaram a avaliação do equilíbrio postural. A avaliação iniciou-se na plataforma de força e em seguida foram realizados os testes funcionais (de forma aleatória). Todas as participantes foram familiarizadas com os equipamentos e o protocolo experimental antes do início da avaliação.

Plataforma de Força: As atletas foram posicionadas sobre a plataforma de forma (BIOMEC400, EMG System do Brasil, SP Ltda.) em apoio unipodal (membro dominante) e descalças. O teste de equilíbrio consistiu em três tentativas de 30 segundos (s) cada, com $30 \mathrm{~s}$ de intervalo entre cada tentativa ${ }^{13}$. Durante os testes, o membro contralateral permanecia flexionado (aproximadamente $90^{\circ}$ de flexão de joelho, quadril em posição neutra), os membros superiores ao longo do corpo e o olhar fixo na linha do horizonte em direção ao alvo posicionado a 2,0 metros de distância (figura 1). A média das três tentativas foi selecionada para a análise.

A força vertical de reação do solo sobre a plataforma foi derivada de uma amostragem de $100 \mathrm{~Hz}$. Todos os sinais de força registrados foram filtrados com um filtro de banda-passante baixo de $35-\mathrm{Hz}$ e de segunda ordem (Butterworth filter) para eliminar os ruídos elétricos. Para aquisição e tratamento dos parâmetros de equilíbrio, foi utilizado o próprio software Bioanalysis da plataforma BIOMEC400, o qual é compilado com rotinas de computação de análises MATLAB (The Mathworks, Natick, MA). Os principais parâmetros de equilíbrio baseados no COP foram computados: A área de elipse do COP (A-COP em $\mathrm{cm}^{2}$ ) e a velocidade média de oscilação do COP (VEL em $\left.\mathrm{cm} / \mathrm{s}\right)$ foram determinadas nas direções: anteroposterior (A/P) e médio-lateral (M/L). A validade e a confiabilidade dos parâmetros de equilíbrio calculados com esta plataforma têm sido evidenciadas por um estudo prévio ${ }^{14}$.

Testes funcionais de equilíbrio: Foram realizados três testes funcio- 
nais para avaliação do equilíbrio postural: 1) Star Excursion Balance Test (SEBT), 2) Saltos laterais e 3) Figura em oito, como descritos a seguirA ordem dos testes foi aleatória, por sorteio simples.

O teste SEBT modificado foi realizado como descrito por Pliky et al. ${ }^{15}$. Os participantes permaneciam com o pé de apoio centralizado a intersecção das linhas (anterior, posterolateral e posteromedial). A posição em apoio unipodal foi mantida enquanto o membro inferior contralateral alcançava a maior distância possível sobre as linhas (fitas métricas) e o valor era medido pelo avaliador (figura 1A). Foram realizadas três tentativas para cada direção. As mesmas instruções foram dadas a todos os sujeitos. Caso o pé de apoio fosse movido, o teste era interrompido, posicionava-se novamente o pé do sujeito no local indicado e repetia-se o teste. A pontuação do SEBT foi calculada dividindo a soma das distâncias máximas alcançadas (em centímetros) nas três direções pelo comprimento real do membro inferior (LL) avaliado, e por fim multiplicado por três. Os valores finais foram então expressos em porcentagem multiplicando-se por 100, como segue na equação proposta por Filipa et al. ${ }^{7}$, sendo a média de três tentativas utilizada como score final:

\section{$S E B T=(A+P M+P L) /(L L \times 3) \times 100$}

Para o teste de Salto lateral (Side Hop Test) ${ }^{16}$, as atletas foram orientadas a saltar lateralmente, em apoio unipodal, em uma distância de 30 centímetros. Uma repetição consistia em saltar lateralmente $30 \mathrm{~cm}$ e retornar ao ponto inicial. Foram realizadas 10 repetições consecutivas e as atletas eram orientadas a saltar o mais rápido possível (figura 1B). Para o cálculo dos resultados, utilizou-se a média do tempo para as três tentativas, em segundos.

Para o teste da Figura em Oito (Figure of eight hop test) ${ }^{15,16}$, foi utilizada uma pista com cinco metros de comprimento, delimitada por cones. As atletas foram orientadas a realizar duas voltas no percurso, em apoio unipodal, o mais rápido possível (figura $1 \mathrm{C}$ ). Os resultados foram calculados utilizando a média do tempo das três tentativas, em segundos.

\section{Análise de Dados}

Todas as variáveis foram testadas quanto à distribuição normal dos dados por meio do teste de Shapiro-Wilk. Após confirmar a distribuição normal dos dados, as correlações entre as medidas dos testes funcionais e os parâmetros de equilíbrio da plataforma foram desenvolvidas

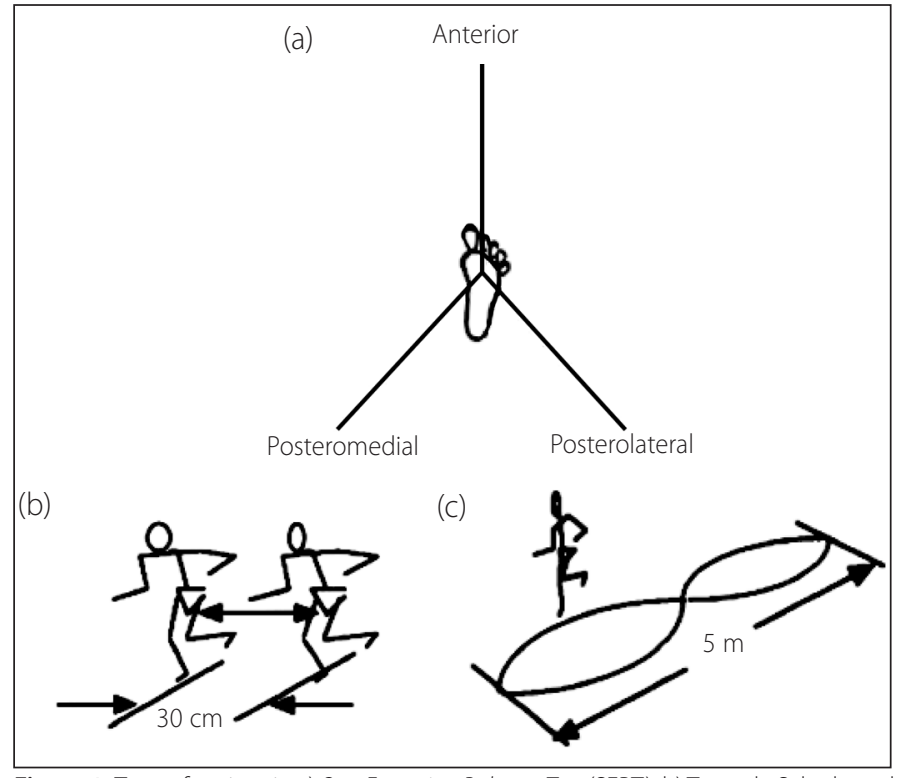

Figura 1. Testes funcionais. a) Star Excursion Balance Test (SEBT); b) Teste de Salto lateral; c) Teste da Figura em Oito. conforme o teste de correlação de Pearson. Todas as análises foram realizadas com o pacote estatístico SPSS (v.15) a significância adotada foi de $5 \%(P<0,05)$. Os resultados descritivos foram apresentados em média e desvio padrão (DP).

\section{RESULTADOS}

As características antropométricas das participantes do estudo como idade, peso, altura, índice de massa corporal (IMC) e tempo de pratica estão apresentados de maneira descritiva, na qual, os valores em média foram: idade de 15 anos ( $D P=1,24)$, peso de $62 \mathrm{~kg}(\mathrm{DP}=9,30)$, altura de 1,66 m (DP=0,05), IMC de $22 \mathrm{~kg} / \mathrm{m}^{2}(\mathrm{DP}=2,77)$.

O tempo de prática esportiva foi em média de quatro anos. Nenhuma atleta apresentou histórico de lesões prévias no membro avaliado. Os resultados obtidos nos testes funcionais como teste da figura em oito, teste de Salto lateral e Star Excursion Balance Test (SEBT) e os parâmetros de equilíbrio da plataforma de força estão apresentados na tabela 1.

Os resultados da correlação entre os testes funcionais (Figura em Oito, salto lateral e SEBT) e os parâmetros da plataforma de força (VEL A/P, VEL M/L e A-COP) estão apresentados na tabela 2. Em geral, as correlações entre os testes funcionais e a plataforma de força foram de fraca a moderada (r 0.01 a -0.69), dependendo da variável analisada. As melhores correlações foram encontradas entre o teste SEBT e o parâmetro A-COP da plataforma (tabela 2), sendo uma correlação moderada e significativa $(r=-0.69)$, indicando que quanto maior a distância alcançada no teste melhor foi o equilíbrio postural sobre a plataforma de força (menores valores da A-COP). Por seguinte, os resultados entre o teste da figura em oito e A-COP apresentaram uma correção de fraca a moderada, como por exemplo, um $r=0.43$ para a variável A-COP, indicando que quanto maior o tempo de realização do teste maior é a instabilidade postural. Os demais resultados, especificamente entre os testes funcionais e os parâmetros de velocidade (VEL) em ambas as direções não foram significantes ( $r=0.01$ a 0.36).

Tabela 1. Análise descritiva dos dados obtidos nos testes funcionais e na plataforma de força.

\begin{tabular}{c|c|c}
\hline Variáveis & Média & Desvio Padrão (DP) \\
\hline Figura em Oito (segundos) & 13.96 & $(2.11)$ \\
\hline Salto lateral (segundos) & 6.06 & $(1.75)$ \\
\hline SEBT (\%) & 86.05 & $(5.68)$ \\
\hline A-COP $\left(\mathrm{cm}^{2}\right)$ & 6.62 & $(2.26)$ \\
\hline VEL A/P $(\mathrm{cm} / \mathrm{s})$ & 2.24 & $(0.49)$ \\
\hline VEL M/L $(\mathrm{cm} / \mathrm{s})$ & 2.45 & $(0.56)$ \\
\hline
\end{tabular}

Figura em Oito: Teste da Figura em 8; Salto lateral: Teste de salto lateral; SEBT: Star Excursion Balance test; A-COP: Área de deslocamento do centro de pressão; VEL A/P: velocidade de deslocamento do centro de pressão no sentido anteroposterior: VEL M/L: velocidade de deslocamento do centro de pressão no sentido médio-lateral.

Tabela 2. Correlações $r$ de Pearson entre os testes funcionais e as medidas da plataforma de força.

\begin{tabular}{c|c|c|c}
\hline & VEL A/P & VEL M/L & A-COP \\
\hline $\begin{array}{c}\text { Figura em Oito } \\
\text { (segundos) }\end{array}$ & $0.18(0.510)$ & $0.07(0.800)$ & $0.43(0.100)$ \\
\hline $\begin{array}{c}\text { Salto lateral (segun- } \\
\text { dos) }\end{array}$ & $0.16(0.556)$ & $0.23(0.401)$ & $0.27(0.325)$ \\
\hline SEBT (\%) & $0.01(0.960)$ & $0.36(0.181)$ & $-0.69(0.012)^{*}$ \\
\hline
\end{tabular}

Correlações de Pearson: $r$. *Significância ( $p$ < 0.05). Figura em Oito: Teste da figura em Oito; Salto lateral: Teste de salto lateral; SEBT: Star Excursion Balance test; A-COP: Área de deslocamento do centro de pressão; VEL A/P: velocidade de deslocamento do centro de pressão no sentido anteroposterior: VEL M/L: velocidade de deslocamento do centro de pressão no sentido médio-lateral. 


\section{DISCUSSÃO}

Os resultados mostram que houve uma correlação de fraca a moderada entre os testes funcionais e a plataforma de força, onde a melhor correlação encontrada foi entre o teste SEBT e o parâmetro A-COP. Este resultado indica que quanto maior a distância alcançada no teste SEBT, menor é a instabilidade postural sobre a plataforma. Outro achado interessante foi no teste da figura em oito, que apresentou uma correlação moderada com o parâmetro A-COP, indicando que quanto menor o tempo de realização do teste, maior é a instabilidade postural.

Até o presente momento, este é o primeiro estudo que incluiu três principais testes funcionais de estabilidade articular para determinar a relação deles com os parâmetros COP de uma plataforma de força durante um teste de equilíbrio unipodal em atletas. Neste sentido, a discussão dos resultados se torna difícil em relação a uma comparação direta com resultados da literatura. Entretanto, Trojian et al. ${ }^{17}$ que avaliaram o desempenho de atletas durante o teste funcional Single Leg Balance, demonstraram que os atletas com piores resultados tinham mais chances de sofrer lesões de tornozelo pela instabilidade postural. McGuine et al, ${ }^{18}$ apontaram o poder preditivo das medidas da plataforma de força nas entorses de tornozelo em jogadores juvenis de basquetebol. Recentemente, Dallinga et al. ${ }^{19}$ realizaram uma revisão sistemática com intuito de encontrar ferramentas que poderiam prever o risco de lesões em membros inferiores nos esportes de equipe. Esses autores constaram que o teste funcional SEBT apresenta uma sensibilidade apurada para discriminar adolescentes do sexo feminino a desenvolverem futuras lesões. Outros estudos mostraram ainda que o SEBT apresenta boa sensibilidade para indicar déficits posturais ${ }^{20,21}$, o que corrobora com os resultados do presente estudo nas correlações com o parâmetro A-COP. Este parâmetro identifica o deslocamento do COP em uma área correspondendo às direções anteroposterior e médio-lateral para manter o centro de massa do corpo dentro da base de sustentação ${ }^{4}$. Embora o equilíbrio seja multifacetado, a base do controle postural para manutenção do equilíbrio ocorre por meio das estratégias sensório-motoras e biomecânicas, caracterizando uma grande importância identificar testes mais simples e práticos que poderiam de alguma forma revelar esse fenômeno entre indivíduos diferentes.

É importante ressaltar que somente as medidas de uma plataforma de força, com base no comportamento do COP, podem identificar os déficits de equilíbrio associados aos mecanismos neuromusculares e biomecânicos ${ }^{12,22}$. A correlação apresentada no estudo auxilia a compreensão para identificar quais dos testes funcionais poderiam estar mais associados às medidas da plataforma e assim direcionar as tomadas de decisões clínicas quanto às avaliações do equilíbrio postural na prevenção e reabilitação de atletas. Por outro lado, os resultados deste estudo sugerem que nem todos os testes funcionais fornecem a mesma informação quanto aos mecanismos de controle postural como recentemente apontado por Nguyen ${ }^{12}$, devido aos valores encontrados nas correlações fracas e não significantes, com exceção para o SEBT.

Por fim, os resultados deste estudo não podem ser generalizados para atletas de modalidades diferentes do futsal e handebol e tão pouco para atletas do sexo masculino. Neste estudo, o direcionamento da avaliação foi de maneira semi-estática (unipodal) na plataforma de força, o que limita a conclusão dos resultados para situações dinâmicas como as correlações com o teste da figura em oito que possui uma característica não estática. Novos trabalhos envolvendo um maior número da amostra bem como indivíduos atletas de maior idade (acima de 18 anos) são necessários para compreender melhor a relação entres esses testes funcionais e as medidas da plataforma de força.

\section{CONCLUSÃO}

Os resultados suportam a ideia de que alguns testes funcionais não apresentam, necessariamente, a mesma informação que a plataforma de força fornece quanto aos mecanismos de controle postural para equilíbrio. Entretanto, o teste SEBT poderia ser utilizado com moderada relevância na avaliação da estabilidade postural em atletas por apresentar melhor correlação com um dos principais parâmetros da plataforma de força, a área do COP. Este estudo colabora para a avaliação do equilíbrio em atletas e sugere que alguns testes funcionais de estabilidade articular/postural deveriam ser usados com maior cautela para identificação dos possíveis déficits de equilíbrio, especialmente quando usados para avaliação clínica.

Todos os autores declararam não haver qualquer potencial conflito de interesses referente a este artigo.

\section{REFERÊNCIAS}

1. Silva T, Ribeiro F, Venâncio J. Comparação da performance funcional do membro inferior entre jovens futebolistas e jovens não treinados. Fisioter. Mov. 2010;23(1):105-12.

2. Gazzola JM, Perracini MR, Ganançça MM. Functional balance associated factors in the eldery with chronic vestibular disorder. Braz J Otorhinolaryngol. 2006;72(5): 683-90.

3. Nashner L. Pratical biomechanics and physiology of balance. In: Jacobson G, Newman C, Kartush J. eds. Handbook of balance function and testing. St. Louis: Mosby Year Book; 1993. p.261-79.

4. Winter DA, Patla AE, Ishac M, Gage WH. Motor mechanism of balance during quiet standing. J Electromyogr Kinesiol. 2003;13: 49-56.

5. Kinzey SJ, Armstrong CW. The reliability of the star-excursion test in assessing dynamic balance. J Orthop Sports Phys Ther. 1998:27:356-60.

6. Howe TE, Rochester L, Jackson A, Banks PMH, Blair VA. Exercise for improving balance in older people (review). Cochrane Database of Systematic Reviews 2009;1-150.

7. Filipa A, Byrnes R, Paterno MV, Myer GD, Hewett TE. Neuromuscular training improves performance on the star excursion balance test in young female athletes. journal of orthopedic \& sports physical therapy. 2010; 40:9.

8. Nidhi S, Archna S, Jaspal SS. Functional performance testing in athletes with functional ankle instability Asian Journal of Sports Medicine. 2011;2(4):249-58.

9. Winter DA. Human balance and posture control during standing and walking. Gait Posture. 1995; 3:193-214.

10. Schimid M, Conforto S. Stability limits in the assessment of postural control through the Time-to-Boundary function. Proceedings of the 29th Annual International Conference of the IEEE EMBS. August 23-26, 2007

11. Trulsson A, Roos EM, Ageberg E, Garwicz. Relationships between postural orientation and self reported function, hop performance and muscle power in subjects with anterior cruciate ligament injury. BMC Musculoskelet Disord. 2010;1:11:143.

12. Nguyen DT, Kiel DP, Li W, Galica AM, Kang HG, Casey VA, et al. Correlations of clinical and laboratory measures of balance in older men and women. Arth Care Res. 2012;64(12):1895-902.

13. Shigaki L. Rabello LM, Camargo MZ et al. Análise comparativa do equilíbrio Unipodal de atletas de Ginástica Rítmica. Rev Bras Med Esporte. 2013;19(2):104-7.

14. da Silva RA, Martin B, Parreira RB, Teixeira DC, Amorim CF. Age-related differences in time-limit performance and force platform-based balance measures during one-leg stance. J Electromyogr Kinesiol. 2013, 23:634-9.

15. Plisky PJ, Rauh MJ, Kaminski TW, Underwood FB. Star excursion balance test as a predictor of lower extremity injury in high school basketball players. J Orthop Sports Phys Ther. 2006;36:911-9.

16. Caffrey E, Docherty CL, Schrader J, Klossner J. The ability of 4 single-limb hopping the functional performance deficits in individuals with functional ankle instability. J Orthop Sports phys Ther. 2009;39:799-806

17. Trojian TH, McKeag DB. Single leg balance test to identify risk of ankle sprains. Br J Sports Med. 2006:40:610-3.

18. McGuine TA, Greene JJ, Best T, Leverson G. Abstract balance as a predictor of ankle injuries in high school basketball players. Clin J Sport Med. 2000;10:239-44.

19. Dallinga JM, Benjaminse A, Lemmink KA. Which screening tools can predict injury to the lower extremities in team sports?: a systematic review. Sports Med. 2012;1;42(9):791-815.

20. Gribble PA, Hertel J, Denegar CR. Chronic ankle instability and fatigue create proximal joint alterations during performance of the star excursion balance test. Int J Sports Med. 2007;28:236-42.

21. Hertel J, Braham RA, Hale SA, Olmsted-Kramer LC. Simplifying the star excursion balance test: analyses of subjects with and without chronic ankle instability. J Orthop Sports Phys Ther. 2006;36:131-7.

22. Mancini M, Horak FB. The relevance of clinical balance assessment tools to differentiate balance deficits. Eur.J Phys Rehabil Med. 2010;46 (2):239-48. 